\title{
The Neuro Image Quiz, Answers
}

\section{\begin{tabular}{l|l|l} 
T.P. & Thomas P. & Naidich \\
\hline
\end{tabular}}

Thomas P. Naidich, Department of Radiology, Children's Memorial Hospital, Northwestern University Medical School, Chicago, IL 60614 (USA)

This infant has diastematomyelia, defined as a sagittal clefting of the spinal cord into two, not necessarily symmetrical 'hemicords', each lined by its own pia mater. The relationships of the hemicords to the arach-noidal and dural tubes define two different forms of diastematomyelia [1,7]:

(1) In approximately $50 \%$ of all cases of di astematomyelia, the two pia-lined hemicords lie side by side in a single undivided subarachnoid space and are encompassed within a single undivided tube of arach noid mater. External to the arachnoid tube lies a single co-axial tube of dura. This form of diastematomyelia is not associated with any bone spur or fibrous septum.

(2) In the other $50 \%$ of cases of diastematomyelia, as in this case, the arachnoid mater and dura mater also separate into paired arachnoidal and dural tubes, one meningeal tube surrounding each hemicord. The medial walls of the two dural tubes then form a double layer of dura that lies between the two hemicords. This double layer of dura is called the dural or fibrous septum. Most patients with this type of diastematomyelia have an osteocartilaginous spur. In these cases the spur passes be tween the two dural tubes, medial and external to the dural tubes. The spur does not invade through neural tissue.

Diastematomyelia occurs very much more commonly in females. In specific series, females constituted from $75[1,5]$ to $78,[3,8]$ to $94 \%[2]$ of the entire series.

Cutaneous stigmata are found in 50-75\% of patients with diastematomyelia [1,3-5, 8]. The most common stigmata are hypertrichosis (38\%), skin dimples and sinuses $(13 \%)$, non-hairy nevi $(10 \%)$, and lipomas $(6 \%)$. Hemangiomas and meningoceles are rare $(1.3 \%$ each).

The incidence of diastematomyelia in patients with scoliosis is approximately 6\%. Winter et al. [8] found diastematomyelia in 23 of 392 patients with congenital scoliosis $(5.9 \%)$. Since not all their patients had myelo-grams, they concluded that the true incidence was probably higher.

In patients with diastematomyelia, the incidence of scoliosis is high and increases with patient age. Hilal et al. [2] found the overall incidence of scoliosis was $56 \%$ in 34 patients with diastematomyelia. $44 \%$ had no scoliosis; the average age of this group was 4 years, 5 months. 
$21 \%$ had moderate scoliosis; the average age of this group was 7 years, 7 months. $35 \%$ had severe scoliosis; the average age of this group was 11 years, 1 month. Keim and Greene [5] found scoliosis in $70 \%$ of 20 patients with diastematomyelia. The scoliosis was mild $\left(0-30^{\circ}\right)$ in $21 \%$, moderate $\left(30-60^{\circ}\right)$ in $21 \%$ and severe (more than $60^{\circ}$ ) in $57 \%$. The curvatures observed [5] were a major right thoracic curve $29 \%$, left thoracic curve $21 \%$, left thoraco-lumbar curve $21 \%$, left lumbar curve $14 \%$, mild right thoracic curve $7 \%$ and paralytic curve $7 \%$. Keim and Greene [5] concluded that all scolioses were directly related to structural deformities of the vertebrae and were not neurological in origin (with the single exception of a paralytic curve secondary to poliomyelitis). The spinal column is abnormal in more than $90 \%$ of patients with diastematomyelia. The vertebral bodies show multiple vertebral anomalies in 85 [2] to $95 \%$ [8]. One or more hemivertebrae are seen in approximately 30\% [8]. Failure of segmentation of the bodies with unilateral unsegmented bar or fused vertebral bodies is seen in approximately $56 \%$ [8]. One often sees several short squat vertebral bodies with narrow vertical and sagittal dimensions and narrow intervertebral disc spaces near the level of the bone spur.

The laminae are abnormal in 43 [3] to $91 \%$ [2] of patients. Vertical fusion of adjacent laminae, either ip-silaterally or diagnonally (across the midline), is commonly seen. The bone spur often arises from this inter-segmental laminar fusion.

The laminae are bifid in 87 [3] to $93 \%$ [8] of patients.

The spina bifida often affects multiple levels in continuity or multiple levels above and below a zone of in-tersegmental laminar fusion. When the intersegmental laminar fusion is unilateral, the contralateral laminae may be bifid and everted. The combination of intersegmental laminar fusion and spina bifida is seen in $59 \%$ of patients with diastematomyelia and is highly suggestive of this diagnosis [2].

The spinous process is usually prominent in the area of the bone spur, especially if there is an intersegmental fusion of the adjacent laminae.

The bone spur is identified on plain spine films in $88 \%$ of patients with diastematomyelia (type not speci- 
fied) [8]. Spurs are observed at two or more separate levels in 5 [5] to 8\% [3] of patients. The spur is lumbar in location in 70\% and thoracic in 30\% [8]. The spur may angle forward and upward (43\%), straight forward (29\%) or forward and downward (29\%) [2]. The spur ossifies from one or more separate center(s) in cartilage, so it typically is incomplete in neonates, infants and young children.

The interpediculate distance is widened in the area of the diastematomyelia in 88 [2] to 96 [8] to $100 \%$ [3] of patients. The maximum interpediculate distance is usually at the level of the widest spina bifida and, therefore, is usually a few segments above or below the bone spur [2].

The cleft in the cord may be very short $(1 \mathrm{~mm}$ ) or very extensive (nearly holocord). Keim and Greene [5] reported that the cleft in the cord extended for 2-15 segments (average 5.5 segments) or from 1.5 to $6.8 \mathrm{~cm}$ (average $4.7 \mathrm{~cm}$ ). The dural septum lay at Tl-T6 in 5\%, at T7-T12 in 26\%, at Ll-L3 in 48\% and at L4L5 in $18 \%$. Hilal et al. [2] found the septum was lumbar in $74 \%$ and thoracic in $26 \%$. In the series of Hilal et al. [2], the highest dural partition extended between T3 and T6; the lowest was at SI; patients with severe scoliosis tended to have higher and longer septa [2]. 
Frerebeau, P.; Dimeglio, A.; Gras, M.; Harbi, H.: Diastematomyelia: report of 21 cases surgically treated by a neu-rosurgical and orthopedic team. Child's Brain 10:328-339 (1983).

Hilal, S.K.; Marton, D.; Pollack, E.: Diastematomyelia in children. Radiology 112:609-621 (1974).

Hood, R.W.; Risenborough, E.J.; Nehme, A.-M.; Micheli, L.J.; Strand, R.J.; Neuhauser, E.B.D.: Diastematomyelia and structural spinal deformities. J. Bone J. Surg. 62A: 520-528 (1980).

James, C.C.M.; Lassman, L.P: Diastematomyelia. Archs Dis. Childh. 39:125-130 (1964).

Keim, H.A.; Greene, A.F.: Diastematomyelia and Scoliosis. J. Bone J. Surg. 55A: 1425-1435 (1973).

Naidich, T.P; Harwood-Nash, D.C.: Diastematomyelia: hemicord and meningeal sheaths; single and double arachnoid and dural tubes. Am. J. Neuroradiol. 4: 633-636 (1983).

Naidich, T.P; Harwood-Nash, D.C.; McLone, D.G.: Radiology of spinal dysraphism. Clin. Neurosurg. 30: 341-365 (1983).

Winter, R.B.; Haven, J.J.; Moe, J.H.; Lagaard, S.M.: Diastematomyelia and congenital spine deformities. J. Bone J. Surg. 56A: 27-39 (1974). 